Pacific Journal of Mathematics

HIGHLY TRANSITIVE GROUP ACTIONS ON TREES AND 


\title{
HIGHLY TRANSITIVE GROUP ACTIONS ON TREES AND NORMALIZING TITS SYSTEMS
}

\author{
BENEDICT G. SEIFERT
}

The theory of Tits systems associates to each group $G$ with Tits system a simplicial complex, together with a 'numbering' of its set of vertices, on which the group acts in a highly transitive manner. This numbered simplicial complex is a tree if and only if the Weyl group of the Tits system is an infinite dihedral group, for example when $G$ is $\operatorname{PSL}(2, K), K$ is a local field, with its affine Tits system structure, or when $G$ is the central quotient of the group associated to a Kac-Moody Lie algebra of rank 1. There are non-algebraic examples of such groups as well, such as the full automorphism group of a numbered tree.

In this paper, we investigate the structure of groups acting highly transitively on a tree without preserving a given numbering of the set of vertices. Such groups no longer possess the structure of a Tits system. However, we show that such groups have a pair of subgroups $B$ and $N$ which satisfy all the properties of a Tits system, except the requirement that the generators of the Weyl group should not normalize $B$. We have called a group $G$ with $B$ and $N$ satisfying these properties a normalizing Tits system. We show that these groups have some properties closely related to, but different from, those arising in the theory of Tits systems, such as the structure of the set of 'parabolic subgroups' of $G$. There are very simple examples of such groups, for instance the full group of automorphisms of a tree of $\mathrm{Gl}(2, K), K$ a local field.

The most important property familiar from the theory of Tits systems which still holds for the groups we study here is the existence of a Bruhat decomposition. However, while the Weyl group is still a Coxeter group, with a distinguished set $S$ of generators, the rule of multiplying double cosets by elements of $S$ is very different from the familiar situation: there are elements $s$ in $S$ for which for all $w$ in the Weyl group, s. B.w. B = B.s.w. B.

For the theory of Tits systems and some of its applications, the reader can consult Tits, Bruhat and Tits, Iwahori and Matsumoto and Garland and the bibliographies referred to therein. The idea of studying groups with Bruhat decomposition more general than those with Tits system was first introduced in our work. It was in that paper that the intimate relation between multiple transitivity and groups with Bruhat decomposition was first noticed. But while this paper is thus conceptually closely related to our work, the notion of transitivity here introduced and the proof of Bruhat decomposition are wholly different. Also, the central notion of this paper-that of a normalizing Tits system-is new.

0. Introduction. Trees arise in a variety of mathematical contexts (see [Serre]). They are the simplest examples of affine buildings (for the general theory of buildings see [T], for affine buildings and their origin, 
see [IM], [BT]); it is known, for example, that the buildings associated to $\mathrm{Sl}(2, K)$ where $K$ is a discrete valuation field or to a central quotient of the group associated to a Kac-Moody Lie algebra of rank 2 are trees (see [G], [K]); more generally, the building associated to a group with Tits system is a tree iff its Weyl group is an infinite dihedral group (see [Serre], Ch. 2, §1). It is a general feature of the theory of Tits systems that a group acting on its building preserves a fixed numbering defined on the set of points of the building (see [T]). In the case of a tree, the $B$ and $N$ occurring in the definition of the Tits system are the stabilizers of a link and a maximal totally ordered subgraph containing that link, respectively. It follows from this that a group of automorphisms of a homogeneous tree containing inversions can not give rise to a Tits system with respect to $B$ and $N$ as above. Now, there are many important groups acting on a tree with inversions, for instance the group of all automorphisms or $\operatorname{PGL}(2, K)$, $K$ a local field or, for $K$ any field, $\operatorname{Gl}\left(2, K\left(\left[t, t^{-1}\right]\right)\right)$ acting on the building defined by equivalence classes of $K[t]$-lattices in a free rank 2 module over $K\left[t, t^{-1}\right]$.

It is the purpose of this paper to study an axiomatically defined class of transformation groups, which include these examples. More precisely, we shall suppose that our groups satisfy certain transitivity properties which can be regarded as a natural analogue of multiple transitivity in the context of trees (see $\$ 2$, Definition 2.1). They were motivated by [S.1], where it was shown that a certain geometric notion of multiple transitivity implied properties closely related to Tits systems. But it turns out that the condition we impose is in fact a very direct analogue of Tits' theorem characterizing groups with Tits system in terms of a certain transitivity property of groups acting on buildings (see [T], 3.11, p. 44). Our main result then is that transformation groups with this transitivity properties satisfy an axiom system closely related to Tits systems, which we call 'normalizing Tits systems'. More precisely, we show that $(G, B, N, S)$ where $B$ and $N$ are the stabilizers of a link in the tree and a maximal chain containing it, $W=N / N \cap B$ and $S$ a certain set of generators of $W$, form a normalizing Tits system (see $\S 3$, Definition 3.2). This means essentially that the quadruple $(G, B, N, S)$ satisfies all of Tits' axioms except the condition that no element of $S$ normalizes $B$, which is false for the groups we study. In particular, by contrast to groups with Tits system, $B$ is not self-normalizing.

The group of all automorphisms of a building satisfies our axioms, as do the other examples given above. We should point out that the groups we study here share many properties of groups with Tits systems. For 
instance, $G$ has a Bruhat decomposition (Theorem 2.1.). In fact, as in [S.1], the Bruhat decomposition plays the principal role for these groups. Moreover, we can give an explicit description of the set of 'parabolic' subgroups of such a group (i.e. containing a conjugate of $B$ ) very analogous to (but different from) the description of the set of parabolics in a group with Tits system (Theorem 3.2.). Furthermore we show that there is always a subgroup of index 2 and a set of reflections giving rise to a Tits system (Theorem 3.2.).

There are other intriguing contrasts as well as parallels between Tits systems and normalizing Tits systems. Thus, for the groups here introduced, there exist $s$ in $S$ such that for all $w$ in $W$, B.s.B.w.B = B.s.w.B, which is impossible for groups with Tits system, thus illustrating the crucial role of the axiom B.w.B.s. $\neq B$ of Tits systems in proving the characterization of the set of Weyl group elements (for given $s$ ) for which B.w.B.s. $B=$ B.ws. $B$ in terms of the length function in a Coxeter group.

Finally, we should note that both the result and the proof of this paper are closely related to those discussed in [S.1, S.2]. Indeed, taken together, these results provide evidence for the close relationship between transitivity properties in transformation groups and groups with Bruhat decomposition, and the fact that interesting groups with Bruhat decomposition exist which are very close to groups with Tits systems but do not fit into the framework of Tits' theory.

We have refrained from any reference to the language of buildings, partly to keep this paper self-contained and partly to emphasize the difference to the theory of buildings, where the numbering on the set of points plays a crucial technical role.

The author would like to thank the IHES for its warm hospitality and Ofer Gabber for a useful conversation while preparing this manuscript.

1. Preliminaries. In this paragraph, we establish some basic definitions needed later. By a graph we mean a pair $T=(N, L), N$ a set (called the set of nodes) and $L$ a subset of $N \times N$ (the links of the graph) such that $L$ is invariant under the involution taking $(x, y)$ to $(y, x)$. A tree is a graph $T$ such that

(i) $N$ is connected, i.e. for any $n, n^{\prime}$ there exists a path $n=$ $n(0), \ldots, n^{\prime}=n(m)$, such that $(n(i), n(i+1))$ is in $L . m$ is called the length of the path.

(ii) There exists a unique path as in (i) satisfying, for all $i: n(i) \neq$ $n(i+1), n(i) \neq n(i+2)$. That unique path is called the 'geodesic' from $n$ to $n^{\prime}$. The unique geodesic from $n$ to $n$ is the empty path. 
We denote the geodesic from $n$ to $n^{\prime}$ by $p\left(n, n^{\prime}\right)$. The length of the geodesic from $n$ to $n^{\prime}$ is called the distance from $n$ to $n^{\prime}$, and is denoted by $d\left(n, n^{\prime}\right)$. A tree is said to be homogeneous of valence $v, v$ any cardinal, if and only if the set

$$
N(n)=\left\{n^{\prime} / d\left(n, n^{\prime}\right)=1\right\} \text { has cardinality } v \text {, independently of } n \text {. }
$$

From now on we shall always be dealing with homogeneous trees, of not necessarily finite valence. Such a tree is uniquely defined by its valence (up to isomorphism).

We shall consider any connected (finite or infinite) interval of integers as a graph, by defining $N(I)$ to be $I$ and $L(I)$ the set of pairs of adjacent elements. We shall call such a graph a chain. (A maximal chain, together with a fixed numbering of its set of points in the sense of Tits is an apartment of the tree thought of as an affine building - see [IM] and [T] or [B] for the language of buildings).

For the graph $Z$ formed in this way from the set of integers itself, we shall use the following well known and immediate fact:

LEMMA 1.1. (i) The automorphism group of the graph $Z$, which will be denoted by $W$, is the infinite dihedral group $Z \rtimes[s]$, with $s$ the reflection in $1 / 2$.

(ii) $W$ acts freely and transitively on $L(Z)$.

A graph embedding of a subgraph of $Z$ in $T$ will be called a flag in $T$. We denote the set of flags of type $I$ in $T$ by $F(I)$. Furthermore, given $J$ contained in $I$, we shall write $F(J, I)$ as the set of pairs of flags $(f / I, f / J)$ of type $I$ and $J$, respectively, such that $f / J$ is the restriction of $f / I$ to $J$.

The finite oriented flags are just the geodesics.

For a subset $S$ of $N(T)$, we shall denote by $\operatorname{cl}(S)$ the connected component of $S$. If $l$ is a link of $T, n$ a node, then $\operatorname{cl}(l, n)$ will denote the closure of the set consisting of $n$ and of the two nodes of $l$.

2. The Bruhat decomposition. We now define a function from the set of pairs of links in the tree onto the dihedral group $Z \rtimes Z / 2 . Z$, which will provide a notion of relative position. Suppose that $l, l^{\prime}$ are two links of the tree. Let $Z$ be any maximal chain and $f: Z \rightarrow T$ a flag containing both $l$ and $l^{\prime}$ (such flags can easily be shown to exist). According to Lemma 1.1., $f$ is uniquely determined by its image and the link $f((0,1))$. We shall choose $f$ such that $f((0,1))=l$. Then $l^{\prime}=f((m, m \pm 1))$ in $L(Z)$. By Lemma 1.1, again, the pair $(m, m \pm 1)$ corresponds to a unique 
element $w$ of $W=Z \rtimes Z / 2 . Z$. We have thus associated to the pair $\left(l, l^{\prime}\right)$ of links in $T$ an element $w$ in $W$, by means of a flag $f$ for which $f((0,1))=l$. Furthermore, it easy to see that $w$ was independent of $f$, subject to the requirement that $f((0,1))=l$. We denote the map thus defined from $L(T) \times L(T)$ onto $W$ by $c$, and $c^{-1}(w)$ by $R(w)$. We think of $c\left(1,1^{\prime}\right)$ as the 'relative position' of 1 and $1^{\prime}$.

We now define the class of subgroups which we shall consider throughout this paper.

DefinItIon 2.1. A subgroup $G$ acting faithfully on a homogeneous tree is said to be maximally transitive if and only if $G$ acts transitively on the set $F(J, Z)$ of pairs of flags of type $J=(0,1)$ and $Z$ the graph defined by the integers, with the obvious embedding.

LEMMA 2.1. For a maximally transitive group $G, c$ defines a bijection from $(L(T) \times L(T) / G)$ onto $W$.

Proof. The surjectivity of $c$ is immediate. Thus it suffices to show that $G$ acts transitively on each $R(w)=c^{-1}(w)$. For two pairs of links $(l, m)$ and $\left(l^{\prime}, m^{\prime}\right)$ we can choose embeddings into graphs $Z, Z^{\prime}$ isomorphic to the graph defined by the integers which contain $l$ and $m, l^{\prime}$ and $m^{\prime}$, respectively. By hypothesis we can find $g$ in $G$ such that the pair $(l, Z)$ is mapped to the pair $\left(l^{\prime}, Z^{\prime}\right)$. Since $m$ and $m^{\prime}$ are uniquely defined as elements of $L(Z), L\left(Z^{\prime}\right)$, respectively, by the relative position vis-a-vis $l$ and $l^{\prime}, m$ will be mapped to $m^{\prime}$, and thus $g .(l, m)=\left(l^{\prime}, m^{\prime}\right)$.

We now fix a maximally transitive group $G$ and a maximal flag $f: Z \rightarrow T$. We shall sometimes identify $Z$ with $f(Z)$, and write $m$, $(m, m+1)$, for $f(m), f((m, m+1))$, etc. We let $N$ be the subgroup of $G$ leaving $f(Z)$ invariant and $B$ the stabilizer of the link $f((0,1))$. We shall prove that $G$ has Bruhat decomposition with respect to $B$ and $N$, i.e. that the following result holds:

THEOREM 2.2. (i) $B \cap N$ is normal in $N$. Letting $W$ be the quotient $N / N \cap B$, we have

(ii) $G=\amalg_{w \in W} B \cdot w \cdot B$.

Proof. We first note that, by Definition 2.1., $N$ acts transitively on the set of links in the subgraph $Z$. The restriction of $N$ to $Z$ defines an exact sequence

$$
1 \rightarrow H \rightarrow N \stackrel{\pi}{\rightarrow} W \rightarrow 1
$$


with $W$ still acting transitively on $L(Z)$. Since $W$ acts faithfully on $f(Z)$, Lemma 1.1. implies that it also acts freely on $L(f(Z))$. Acting transitively and freely on $L(f(Z)$ ), again by Lemma 1.1., $W$ is the full automorphism group of the graph $f(Z)$, i.e. it is the dihedral group $\mathbf{Z} \rtimes \mathbf{Z} / 2 \mathbf{Z}$.

$B \cap N$ is the stabilizer of $(0,1)$ in $N$; since $W$ acts freely on $L(Z)$, this subgroup coincides with the kernel $H$ in the exact sequence (1); hence it is normal, which proves part (i) of the theorem.

In order to show (ii), we must do the following:

(a) construct a bijection

$$
\rho: B \backslash G / B \rightarrow W
$$

(b) Prove that for $n$ in $N, \rho(B . n . B)=\pi(n)$.

Since $G$ acts transitively on $L(T), L(T)=G / B$ and hence $B \backslash G / B$ $=(L(T) \times L(T) / G$. Applying $c$ and using the Lemma 2.1, we obtain $\rho$ as required, hence (a). Now (b) follows immediately from the definition of the map $c$.

3. The normalizing Tits system. We remind the reader of the definition of a Tits system (or $B N$ pair) (see [T]).

Definition 3.1. Let $G$ be a group, $N$ and $B$ two subgroups, such that

(T.1) the subgroups $B$ and $N$ generate $G$.

(T.2) $B \cap N$ is normal in $N$.

Furthermore, the group $W=N / B \cap N$ has a set $S$ of generators of order 2 such that

(T.3) for all $s$ in $S$ and for any choice of coset representatives $n(s)$ of $s$ in $N, n(s)$ does not normalize $B$.

(T.4) for each $w$ in $W, s$ in $S$, the following inclusion holds:

$$
\text { B.w.B.s. } B \subseteq B . w s . B \cup B . w . B
$$

where we write, as usual, $B . w=B . n(w)$ for some $n(w)$ projecting to $w$

A quadruple $(G, B, N, S)$ satisfying these conditions is said to be a Tits system (or $B N$ pair in the terminology of [T]). 
Definition 3.2. $(G, B, N, S)$ as above is said to be a normalizing Tits system if and only if it satisfies the axioms above with condition (T.3) replaced by the condition

(T'.3) There exists $s$ in $S$ such that $n(s)$ normalizes $B$.

We now let $G$ be a group acting faithfully and maximally transitively (see Definition 2.1.) on a homogeneous tree, $f$ a fixed flag, and let $B, N$ and $W$ be defined in terms of $f$ as in $\S 2$, and denote by $(n, n+1)$, etc. the elements in $L(T)$ defined by this embedding. We shall denote by $l$ the element $(0,1)$ in $L(Z)$ embedded in $L(T)$. In particular, $W$ is the automorphism group of the integers thought of as a graph. For each $i$ in $1 / 2 . Z$ we let $s_{i}$ be the reflection in $i$. We let $S=\left\{s_{0}, s_{1 / 2}\right\}$. It is well known that $(W, S)$ is a Coxeter system [B, ch. 4].

THEOREM 3.1. The quadruple $(G, B, N, S)$, forms a normalizing Tits system. Furthermore, there exists no $S^{\prime} \subseteq W$ such that $\left(G, B, N, S^{\prime}\right)$ is a Tits system.

Proof. From Bruhat decomposition for the pair $(B, N)$ we know (T.1) and (T.2).

We now prove that $(G, B, N, S)$ satisfies ( $\left.\mathrm{T}^{\prime} .3\right)$. Let $(a, b)$ be in $L(T)$. Then it is immediate that $B$ coincides with the stabilizer of $(a, b)$ if and only if $(a, b)=(0,1)$ or $(1,0)$. Hence, the normalizer of $B$ in $G$ consists of the set of elements in $G$ which preserve the set $\{0,1\}$. In particular, if $n$ is an arbitrary element in $N$ projecting to $s_{1 / 2}$, then $n$ normalizes $B$, and $\langle B, n\rangle=B \cup B . n(s)$. This proves that $\left(\mathrm{T}^{\prime} .3\right)$ is verified.

It remains to prove (T.4). As in $\S 2, R(w)$ will denote the $G$-orbit in $L(T) \times L(T)$ indexed by $w$.

Suppose now that $w_{1}, w_{2}$ are two elements of $W$. We are interested in the subset $W\left(w_{1}, w_{2}\right)$ defined as follows:

DEFINITION 3.3.

$$
B \cdot w_{1} \cdot B \cdot w_{2} \cdot B=\coprod_{W\left(w_{1}, w_{2}\right)} B \cdot w \cdot B .
$$

By Bruhat decomposition, any minimal $B$-bi-invariant subset of $G$ is clearly of the form B.w.B. Hence it is clear that the left-hand side in Definition 3.3., being $B$-bi-invariant, must be a disjoint union of such double cosets, and hence is of the form indicated. We wish to characterize the set $W\left(w_{1}, w_{2}\right)$ geometrically. 
Definition 3.4. A triple $\left(l, l^{\prime}, l^{\prime \prime}\right)$ of elements in $L(T)$ is said to be of type $\left(w_{1}, w_{2}, w\right)$ iff $\left(l, l^{\prime}\right) \in R\left(w_{1}\right),\left(l^{\prime}, l^{\prime \prime}\right) \in R\left(w_{2}\right)$, and $\left(1,1^{\prime \prime}\right) \in R(w)$. For $w_{1}, w_{2}$, we let

$\Delta\left(w_{1}, w_{2}\right)=\left\{w /\right.$ there exists a triple in $L(T)$ of type $\left.\left(w_{1}, w_{2}, w\right)\right\}$.

Proposition 3.1. For any $w_{1}, w_{2}$,

$$
W\left(w_{1}, w_{2}\right)=\Delta\left(w_{1}, w_{2}\right) .
$$

Proof. (i) Suppose $w \in \Delta\left(w_{1}, w_{2}\right)$, and let $\left(l, l^{\prime}, l^{\prime \prime}\right)$ be a triple of type $\left(w_{1}, w_{2}, w\right)$.

Then, according to $\S 2$, we can write $l^{\prime}=b^{\prime} . w_{1} . l, l^{\prime \prime}=b^{\prime \prime} . w . l$. Furthermore,

$$
c\left(l^{\prime}, l^{\prime \prime}\right)=c\left(b^{\prime} \cdot w_{1} \cdot 1, b^{\prime \prime} \cdot w \cdot l\right)=w_{2} .
$$

By $G$-invariance of $c$, we obtain $c\left(l, w_{1}^{-1} \cdot b^{\prime-1} \cdot b^{\prime \prime} \cdot w \cdot l\right)=w_{2}$. Hence, again by $\S 2$, we can write (modulo $B$ ) the element $g=w_{1}^{-1} \cdot b^{-1} \cdot b^{\prime \prime} \cdot w=b^{\prime \prime \prime} \cdot w_{2}$, and hence $b^{\prime \prime} \cdot w=b^{\prime} \cdot w_{1} \cdot b^{\prime \prime \prime} \cdot w_{2}(\bmod B$ on the right $)$, which is the result required.

(ii) Conversely, suppose $w$ in $W\left(w_{1}, w_{2}\right)$. Then we can write $b . w . l=$ $b_{1} \cdot w_{1} \cdot b_{2} \cdot w_{2} \cdot l$. Letting $l^{\prime}=b_{1} w_{1} \cdot l$ and $l^{\prime \prime}=b . w . l$, we see that $c\left(l, l^{\prime}\right)=w_{1}$, $c\left(l, l^{\prime \prime}\right)=w$. Furthermore,

$$
c\left(l^{\prime}, l^{\prime \prime}\right)=c\left(b_{1} \cdot w_{1} \cdot l, b_{1} \cdot w_{1} \cdot b_{2} \cdot w_{2} \cdot l\right)=c\left(l, b_{2} \cdot w_{2} \cdot l\right)=w_{2} .
$$

Hence $\left(l, l^{\prime}, l^{\prime \prime}\right)$ is a triangle of type $\left(w_{1}, w_{2}, w\right)$, as required.

To complete the proof of Theorem 3.1., it suffices to characterize, for $s_{i}, i=1 / 2$ or 0 , and for $w \in W$, the set $\Delta\left(w, s_{i}\right)$. In fact, in view of the Proposition 3.1, (T.4) is clearly a consequence of the following result:

LEMMA 3.1. (i) $\Delta\left(w, s_{0}\right)=\left\{w, s_{0} . w\right\}$.

(ii) $\Delta\left(w, s_{1 / 2}\right)=\left\{s_{1 / 2} \cdot w\right\}$.

We shall prove a more general result about $\Delta(w, v)$ of which this is a special case and which will be used later on.

First, we observe that $w=w_{1} \cdot w_{2}$ is in $\Delta\left(w_{1}, w_{2}\right)$. Indeed, fixing a common apartment $Z$ containing $l, l^{\prime}=w_{1} \cdot l, l^{\prime \prime}=w_{1} \cdot w_{2} \cdot l$, and using the invariance of $c$, it is clear that $\left(l, l^{\prime}, l^{\prime \prime}\right)$ is a triple of type $\left(w_{1}, w_{2}, w_{1} \cdot w_{2}\right)$. Given two links $l, l^{\prime}$ we define the interval between $l$ and $l^{\prime}$ as follows:

$$
\left[1,1^{\prime}\right]=\left\{\begin{array}{l}
\phi \text { if } 1 \text { and } 1^{\prime} \text { coincide as sets; } \\
\text { the smallest integral interval }[a, b], \text { for which } a \leq b \\
\text { are integers of which one belongs to } 1 \text { and the other } \\
\text { to } 1^{\prime}, \text { otherwise. }
\end{array}\right.
$$


Proposition 3.2. $\Delta\left(w_{1}, w_{2}\right)=\left\{w_{1} \cdot w_{2}, r_{p} \cdot w_{1} \cdot w_{2} / r_{p}\right.$ is the reflection in the point $p$, where $p$ ranges over the set $\left.P=\left[1,1^{\prime \prime}\right] \cap\left[l^{\prime}, l^{\prime \prime}\right]\right\}$.

Proof. We choose the triple $\left(l, l^{\prime}, l^{\prime \prime}\right)$ of type $\left(w_{1}, w_{2}, w\right)$ (where $w=$ $w_{1} \cdot w_{2}$ ) lying in a fixed apartment $Z$. Suppose $p$ is in $P$. We choose an apartment $Z^{\prime}$ such that $Z \cap Z^{\prime}=\operatorname{cl}\left(p, l^{\prime}\right)$. It follows from $\operatorname{val}(T)>2$ that such a $Z^{\prime}$ exists. Let $m^{\prime \prime}$ be the unique element in $L\left(Z^{\prime}\right)$ such that $\left(l^{\prime}, m^{\prime \prime}\right)$ is in $R\left(w_{2}\right)$. Clearly $m^{\prime \prime}=r_{p}\left(1^{\prime \prime}\right)$. Let $Z^{\prime \prime}$ be any apartment containing both $l$, and $m^{\prime \prime}$. It is easily verified that $Z^{\prime \prime}$ contains $l^{\prime \prime}$. Since $l, l^{\prime \prime}, m^{\prime \prime}$ all lie in $Z^{\prime \prime}$ and $\left(l, l^{\prime \prime}\right) \in R(w),\left(1^{\prime \prime}, m^{\prime \prime}\right) \in R\left(r_{\rho}\right), m^{\prime \prime}=r_{p} \cdot w(l)$.

On the other hand, suppose $\left(l, l^{\prime}, m^{\prime \prime}\right)$ is a triple of type $\left(w_{1}, w_{2}, w\right)$, with $m^{\prime \prime}$ not in $Z$ (the apartment containing $l$ and $l^{\prime}$ ), and let $p$ be the unique point of $Z$ of minimal distance to $m^{\prime \prime}$. Suppose $g, g^{\prime}$ are geodesics from 1 and $1^{\prime}$, respectively to $m^{\prime \prime}$. Both of them contain a link one of whose points is $p$. One sees easily that if $p$ is not in $P$, then one can choose $Z^{\prime}$ containing $l, l^{\prime}, m^{\prime \prime}$, and hence $u=w$. If, on the other hand, $p$ is in $P$, then choose $Z^{\prime}$ and $Z^{\prime \prime}$ as above. We can then repeat the argument above to see that $\left(l, m^{\prime \prime}\right) \in R\left(r_{\rho} \cdot w\right)$. This proves the proposition.

We shall use it in the following slightly more convenient form, which uses the fact that the product of reflections in $i$ and $i+1$ is translation by 2 in the Weyl group:

CoRollary 3.1. $\Delta\left(w_{1}, w_{2}\right)=\left\{w, s . w, d . s . w, \ldots d^{r-1}\right.$. s.w $\}$, where $w=$ $w_{1} \cdot w_{2}, r=\#(P), s=s_{0}$ or $s_{1}$, and d translation by 2 in the Weyl group.

We now prove Lemma 3.1, and hence (T.4) from Proposition 3.2. First, if $s=s_{1 / 2}$ then $\left[1,1^{\prime}\right]$ is empty, and hence $\Delta(s, w)=\{s . w\}$. If $s=s_{1}$ then $\left[1,1^{\prime}\right]=\{1\}$. Hence,

$$
\Delta(s, w)= \begin{cases}\{s . w, w\} & \text { if } 1 \in\left[1^{\prime}, 1^{\prime \prime}\right] \\ \{s . w\} & \text { otherwise. }\end{cases}
$$

Notice that there exists $s$, namely $s_{1 / 2}$, for which, for all $w$ in $W$, $\Delta(s . w)=\{s . w\}$, a situation which can not occur for Tits systems.

Finally, it is easy to see that $S$ is the unique set of generators of $W$ for which (T.4) holds. Hence we have proved the last statement of the theorem. 
DeFINITION 3.2. A parabolic subgroup of $G$ is a subgroup containing some conjugate of $B$. We shall denote the set of parabolic subgroups by $\operatorname{Par}(G)$, and those containing $B$ by $\operatorname{Par}(G / B)$.

We wish to describe the set of such parabolics. We use the obvious result

LEMMA 3.2. The map assigning to $P$ the image in $W$ of its intersection with $N$ defines a bijection between $\operatorname{Par}(G / B)$ and the set of subgroups $W^{\prime}$ of $W$, for which, for any $w$ and $v$ in $W^{\prime}$,

$$
B \cdot v \cdot B \cdot w \cdot B \subseteq \coprod_{w^{\prime} \in W^{\prime}} B \cdot w^{\prime} \cdot B,
$$

The lemma follows immediately from Bruhat decomposition.

The set of subgroups of $W$ is very easily listed. Each subgroup of $W$ is of one of the following three types:

(i) $W^{\prime}=n . Z \rtimes Z / 2 . Z$, where $n$ is any positive integer; $W^{\prime}$ is a dihedral group.

(ii) $W^{\prime}=n \cdot Z, n$ a positive integer.

(iii) $W^{\prime}=Z / 2 . Z$

LEMMA 3.3. Suppose $P=B . W^{\prime} . B$ is a parabolic.

Then $W^{\prime}$ is either $Z / 2 . Z$ or else it contains both one of the reflections $s_{0}$ or $s_{1}$ and the subgroup of even translations in $W$.

Proof. We let $h=\max \left\{\#[l, w(l)] / w \in W^{\prime}\right\}$, where $l=(0,1)$. We distinguish three cases:

(i) $h=0$

(ii) $h=1$

(iii) $h>=2$.

Clearly (i) is equivalent to $W^{\prime}$ being either trivial or the group $\left\langle s_{1 / 2}\right\rangle$. If (ii) is the case, then according to Proposition 3.2, $W^{\prime}$ must contain the reflection in at least one of the points 0 or 1 . On the other hand, if $W^{\prime}$ strictly contained the group $\langle s\rangle$, where $s$ is that reflection, then it would have to contain some translation which would immediately imply that in fact $h>1$. Hence (ii) is equivalent to $W^{\prime}=\langle s\rangle, s$ the reflection in one of the points 0 or 1 . On the other hand, $h>=2$ implies, in view of Corollary 3.1. that $W^{\prime}$ contains both the translation by 2 and the reflection in either 0 or 1 . This proves the lemma. 
The proof of Lemma 3.3. allows us to completely characterize the possibilities for $\operatorname{Par}(G / B)$ and consequently for $\operatorname{Par}(G)$. To do this most conveniently, we introduce the following equivalence relation in $N(T)$ :

DEFINITION 3.4. $n$ and $n^{\prime}$ are said to be of equal parity if and only if $d\left(n, n^{\prime}\right)$ is even.

It is immediately verified that in a tree, parity is a $G$-invariant equivalence relation. There are obviously two equivalence classes, and the consequent action of $G$ on the 2-element set of equivalence classes gives rise to an extension $1 \rightarrow G(+) \rightarrow G \rightarrow Z / 2 . Z \rightarrow 1$.

Theorem 3.2(a) $\operatorname{Par}(G / B)=B . W^{\prime} . B$, where $W^{\prime}$ is one of the following

(i) $W^{\prime}=\langle 1\rangle$ and $P=B ; W^{\prime}=W$ and $P=G$.

(ii) $W^{\prime}$ is $=2 . Z \rtimes\left\langle s_{0}\right\rangle=2 . Z \rtimes\left\langle s_{1}\right\rangle$, isomorphic to the infinite dihedral group.

(iii) $W^{\prime}=\langle s\rangle=Z / 2 . Z$, and $s=s_{0}$ or $s_{1}$.

(iv) $W^{\prime}=\left\langle s_{1 / 2}\right\rangle$.

(b) $\operatorname{Par}(G)$ is the set of the following four types of subgroups in $G$ :

(i) $G$ itself and the stabilizers of links in $T$.

(ii) $G(+)$, the subgroup of parity preserving automorphisms of $T$, i.e. preserving the sets $T(+)$ and $T(-)$. Equivalently, the subgroup of $G$ of elements $g$ satisfying, for each $n$ in $N(L), d(n, g . n)$ is even. It is a normal subgroup of index 2 in $G$.

(iii) Stabilizers of points in $N(T)$.

(iv) The subgroups of $G$ preserving a set consisting of a link and its opposite link: they are the normalizers of stabilizers in $G$ of links.

Proof. (a) That (i)-(iv) is an exhaustive list follows from Proposition 3.2 and Corollary 3.1, as in the proof of Lemma 3.3.

(b) Since $G$ acts transitively on $L(T), \operatorname{Par}(G)$ is precisely the set of subgroups containing the stabilizer of a link. Hence it suffices to show that the four types of subgroups in $\operatorname{Par}(G / B)$ are defined by the properties indicated. (i) is obvious.

(ii) We must show that the subgroup $B \cdot W^{\prime} . B$ of (a) (ii) equals $G(+)$. $B$ stabilizes 0 , hence is contained in $G(+) . N^{\prime}$, the inverse image of $W^{\prime}$ in $N$ maps 0 to even elements of $Z$, hence is also contained in $G(+)$. Hence $B . W^{\prime} . B$ is contained in $G(0)$. On the other hand, if $g$ is in $G(+)$, then any inverse image of $w$ in $N$ in any expressions $g=b . w . b^{\prime}$ must lie in $G(+)$, since both $b$ and $b^{\prime}$ are in $B$, hence in $G(+)$. 
(iii) From Bruhat decomposition it follows, as in ii) that for $g=b \cdot w \cdot b^{\prime}$ to stabilizes $i$ it is necessary and sufficient for $w$ to stabilize it. This proves the claim.

(iv) Since the stabilizer of the unordered pair $\{0,1\}$ in $W$ is $s_{1 / 2}$, again Bruhat decomposition implies the characterization given.

\section{REFERENCES}

[B] N. Bourbaki, El. Math., Fasc. XXXIV, Groupes et Algebres de Lie, Chs. 4-6, Masson, Paris, 1981.

[BT] F. Bruhat and J. Tits, Groupes reductifs sur un corps local, Ch. 1, Publ. IHES, No. 41, 1972.

[IM] N. Iwahori and H. Matsumoto, On some Bruhat decomposition and the structure of the Hecke ring of p-adic Chevalley groups, Publ. IHES, Nr. 25 (1965), 5-48.

[G] H. Garland, The arithmetic theory of loop groups, Publ. IHES, 52 (1980).

[K] V. Kac, Infinite Dimensional Lie Algebras, Birkhaeuser, Boston, 1983.

[S.1] B. Seifert, Transitivity properties, Bruhat decomposition and Tits systems, J. L. Math. Soc., 29 (1984), 441-452.

[S.2] _ Transitivity Properties, Bruhat Decomposition and Tits Systems for Transformation Groups II, manuscript, 1984.

[Serre] J. P. Serre, Arbres, Amalgames, SL(2), Asterisque, 461977.

[T] J. Tits, Buildings of Spherical and BN Pairs, Springer Lecture Notes, vol. 386, 1974.

Received April 4, 1984 and in revised form September 1, 1985. The author would like to express his gratitude to the Institute des Hautes Etudes Scientifiques for its warm hospitality while this research was being done. He would also like to thank Ofer Gaber for useful conversations on this topic.

\section{IHES}

91440 BURES-SUR-YVETTE

FRANCE

AND

Corpus Christi College

OXFORD, OX1 4JF, ENGLAND 


\title{
PACIFIC JOURNAL OF MATHEMATICS \\ EDITORS
}

\author{
V. S. VARADARAJAN \\ (Managing Editor) \\ University of California \\ Los Angeles, CA 90024 \\ HERBERT CLEMENS \\ University of Utah \\ Salt Lake City, UT 84112 \\ R. FINN \\ Stanford University \\ Stanford, CA 94305
}

\author{
HERMANN FLASCHKA \\ University of Arizona \\ Tucson, AZ 85721
}

RAMESh A. GANGOLLI

University of Washington Seattle, WA 98195

VAUGHAN F. R. JONES

University of California

Berkeley, CA 94720

ROBION KIRBY

University of California

Berkeley, CA 94720
C. C. MOORE

University of California

Berkeley, CA 94720

H. SAMELSON

Stanford University

Stanford, CA 94305

HAROLD STARK

University of California, San Diego

La Jolla, CA 92093

\section{ASSOCIATE EDITORS}

\author{
R. ARENS \\ E. F. BECKENBACH \\ B. H. NeUmanN \\ F. WOLF \\ K. YOSHIDA \\ (1906-1982)

\section{SUPPORTING INSTITUTIONS} \\ UNIVERSITY OF ARIZONA \\ UNIVERSITY OF BRITISH COLUMBIA \\ CALIFORNIA INSTITUTE OF TECHNOLOGY \\ UNIVERSITY OF CALIFORNIA \\ MONTANA STATE UNIVERSITY \\ UNIVERSITY OF NEVADA, RENO \\ NEW MEXICO STATE UNIVERSITY \\ OREGON STATE UNIVERSITY \\ UNIVERSITY OF OREGON \\ UNIVERSITY OF SOUTHERN CALIFORNIA \\ STANFORD UNIVERSITY \\ UNIVERSITY OF HAWAII \\ UNIVERSITY OF TOKYO \\ UNIVERSITY OF UTAH \\ WASHINGTON STATE UNIVERSITY \\ UNIVERSITY OF WASHINGTON
}

The Supporting Institutions listed above contribute to the cost of publication of this Journal, but they are not owners or publishers and have no responsibility for its content or policies.

Mathematical papers intended for publication in the Pacific Journal of Mathematics should be in typed form or offset-reproduced (not dittoed), double spaced with large margins. Please do not use built up fractions in the text of the manuscript. However, you may use them in the displayed equations. Underline Greek letters in red, German in green, and script in blue. The first paragraph must be capable of being used separately as a synopsis of the entire paper. In particular it should contain no bibliographic references. Please propose a heading for the odd numbered pages of less than 35 characters. Manuscripts, in triplicate, may be sent to any one of the editors. Please classify according to the scheme of Math. Reviews, Index to Vol. 39. Supply name and address of author to whom proofs should be sent. All other communications should be addressed to the managing editor, or Elaine Barth, University of California, Los Angeles, California 90024.

There are page-charges associated with articles appearing in the Pacific Journal of Mathematics. These charges are expected to be paid by the author's University, Government Agency or Company. If the author or authors do not have access to such Institutional support these charges are waived. Single authors will receive 50 free reprints; joint authors will receive a total of 100 free reprints. Additional copies may be obtained at cost in multiples of 50 .

The Pacific Journal of Mathematics is issued monthly as of January 1966. Regular subscription rate: $\$ 190.00$ a year (5 Vols., 10 issues). Special rate: $\$ 95.00$ a year to individual members of supporting institutions.

Subscriptions, orders for numbers issued in the last three calendar years, and changes of address should be sent to Pacific Journal of Mathematics, P.O. Box 969, Carmel Valley, CA 93924, U.S.A. Old back numbers obtainable from Kraus Periodicals Co., Route 100, Millwood, NY 10546.

The Pacific Journal of Mathematics at P.O. Box 969, Carmel Valley, CA 93924 (ISSN 0030-8730) publish'es 5 volumes per year. Application to mail at Second-class postage rates is pending at Carmel Valley, California, and additional mailing offices. Postmaster: send address changes to Pacific Journal of Mathematics, P.O. Box 969, Carmel Valley, CA 93924.

PUBLISHED BY PACIFIC JOURNAL OF MATHEMATICS, A NON-PROFIT CORPORATION

Copyright (C) 1986 by Pacific Journal of Mathematics 


\section{Pacific Journal of Mathematics}

\section{Vol. 125, No. $2 \quad$ October, 1986}

Dale Edward Alspach, On $\mathscr{L}_{p, \lambda}$ spaces for small $\lambda \ldots \ldots \ldots \ldots \ldots \ldots 257$

Jong Sook Bae and Sangsuk Yie, Range of Gateaux differentiable operators

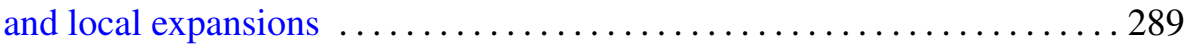

Hubert Berens and Lothar Hetzelt, On accretive operators on $l_{n}^{\infty} \ldots \ldots 301$

Marie-Françoise Bidaut-Véron, Global existence and uniqueness results for singular solutions of the capillarity equation ................. 317

Donald M. Davis and Mark Mahowald, Classification of the stable homotopy types of stunted real projective spaces ................. 335

Aad Dijksma, Heinz K. Langer and Hendrik S. V. de Snoo, Unitary

colligations in $\Pi_{\kappa}$-spaces, characteristic functions and Štraus extensions

Michel Enock and Jean-Marie Schwartz, Algèbres de Kac

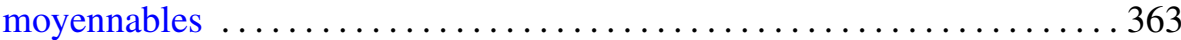

Seppo Granlund, Peter Lindqvist and Olli Martio, Note on the

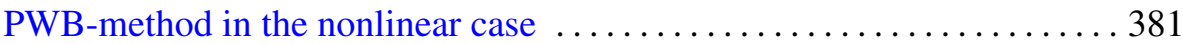

Palle E. T. Jorgensen, Analytic continuation of local representations of Lie

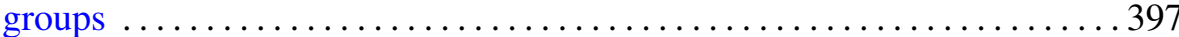

Robert P. Kaufman, Plane curves and removable sets ................409

José M. Montesinos and Wilbur Carrington Whitten, Constructions of two-fold branched covering spaces $\ldots \ldots \ldots \ldots \ldots$.

Benedict Seifert, Highly transitive group actions on trees and normalizing Tits systems

Charles Stuart Stanton, Counting functions and majorization for Jensen

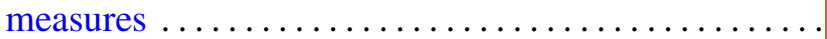

Luen-Fai Tam, On existence criteria for capillary free surfaces without gravity

Zhuocheng Yang, Exposed points of left invariant means 